\title{
Neurovascular Manifestation of Loeys-Dietz Syndrome: A Case Report
}

\author{
Yun-Jeong Lee ${ }^{1+}$, Mi-Sun Yum", Eun-Hee Kim¹, Hae-Won Choi', Beom Hee Lee ${ }^{1,2}$, Han-Wook Yoo, ${ }^{1,2}$ and Tae-Sung Ko* \\ ${ }^{1}$ Department of Pediatrics, ${ }^{2}$ Department of Medical Genetics, Children's Hospital, Asan Medical Center, University of Ulsan College of Medicine, \\ Seoul, Korea
}

\begin{abstract}
Loeys-Dietz syndrome (LDS) is an autosomal dominant disorder caused by heterozygous mutations in the genes encoding transforming growth factor- $\beta$ receptor type 1 or 2 . It is typically characterized by a triad of hypertelorism, cleft palate or bifid uvula, and arterial tortuosity with aneurysm or dissection. Characteristic vascular abnormalities such as tortuosity, aneurysms, dissections, and stenosis are the most severe complications of LDS and can occur in the neurovascular system. We report a 5 -year-old boy who presented with headaches and neurovascular abnormalities and was diagnosed with LDS with a novel mutation of the TGFBR1 gene. It is the first Korean report of neurovascular abnormalities in LDS.
\end{abstract}

Key words: Loeys-Dietz syndrome, Connective tissue disease, Arterial tortuosity, Transforming growth factor- $\beta$ receptor

\section{Introduction}

Loeys-Dietz syndrome (LDS, OMIM 609192) is a connective tissue disease characterized by the triad of hypertelorism, cleft palate or bifid uvula, and arterial tortuosity with aneurysm or dissection. LDS was first described by Loeys in 2005 as an autosomal dominant disorder caused by a heterozygous mutation in the gene encoding transforming growth factor- $\beta$ receptor type 1 (TGFBR1) or type 2 (TGFBR2)."

The most severe abnormality in LDS, and the leading cause of death, is aortic aneurysm and dissection. In LDS, aortic dissection often occurs at a younger age, when aortic diameters are smaller, than in other connective tissue disorders. ${ }^{2,3)}$ The mean onset age of aortic dilatation is in the teens. Due to the aggressive nature of the aortic pathology in LDS, earlysurgical intervention is recommended. ${ }^{3)}$ In addition to the risks of aortic aneurysm, neurovascular involve- ment can cause dissections and intracranial hemorrhages that affect morbidity and mortality. ${ }^{1-3)}$ Here, we report a case of LDS, diagnosed by a novel mutation of TGFBR1 gene, in a patient who presented with headaches at a young age. This suggests the importance of a diagnosis and early intervention in patients with diffuse cerebrovascular dilatation and craniofacial abnormalities typical of LDS.

\section{Case Report}

A 5-year-old boy was referred to our hospital for evaluation of headache, tortuous intracranial arteries and vertebral arteries found up on brain magnetic resonance angiography. The patient's family history was unremarkable for any genetic disease. He was born at 39 weeks of gestation by spontaneous vaginal delivery without complications. His birth weight was 3,580 gm. At 3 years

Received: 25 May 2013, Revised: 17 June 2013, Accepted: 18 June 2013, Published: 30 June 2013

${ }^{*}$ Corresponding author: Tae-Sung Ko, M.D., Ph.D.

Department of Pediatrics, Asan Medical Center, University of Ulsan College of Medicine, 388-1, Poongnap-dong, Songpa-ku, Seoul, 138-736, Korea

Tel: +82-2-3010-3390, Fax: +82-2-473-3725, E-mail: tsko@amc.seoul.kr

these two authors contributed equally to this work.

(c) This is an open-access article distributed under the terms of the Creative Commons Attribution Non-Commercial License (http://creativecommons.org/licenses/by-nc/3.0/) which permits unrestricted non-commercial use, distribution, and reproduction in any medium, provided the original work is properly cited.

(c) Copyright 2013 by the Korean Society of Medical Genetics 
of age, he was diagnosed with an inguinal hernia that was repaired surgically. At 4 years of age, he underwent surgical intervention for exotropia. His weight was $17 \mathrm{~kg}\left(50-70^{\text {th }}\right.$ percentile) and his height was $109.5 \mathrm{~cm}\left(50-70^{\text {th }}\right.$ percentile). His head circumference was $53 \mathrm{~cm}$ ( $97^{\text {th }}$ percentile). On physical examination, bifid uvula, pectus carinatum, joint laxity, and borderline macrocephaly were noted; however, he had no definite facial dysmorphisms except mild hypertelorism and no specific ocular abnormalities. His neurologic examination was normal. For evaluation of the headache, he underwent electroencephalography (EEG) and brain magnetic resonance imaging (MRI) with magnetic resonance angiography (MRA). EEG showed a few generalized slow wave bursts interspersed with sharp wave discharges. Brain MRI/MRA revealed multiple T2 hyperintense lesions in the frontal and parietal subcortical white matter and diffuse, dilated, and tortuous intracranial arteries, including the vertebral artery, the basilar artery, and both the middle and posterior cerebral arteries (Fig. 1). With suspicion of systemic vascular disease, echocardiography and abdomen computed tomography (CT) was performed. Echocardiography showed normal cardiac anatomy but a markedly enlarged aortic sinus and annulus, which measured $50.2 \mathrm{~mm}(\mathrm{Z}$-value=17.99) and 22.8 $\mathrm{mm}$ (Z-value $=8.1$, respectively (Fig. 2). Abdomen CT revealed a prominent celiac trunk and tortuosity of the abdominal aorta (Fig. 3). LDS was suspected based on clinical and imaging findings, including bifid uvula, multiple artery tortuosities, and aneurysm. Molecular genetic testing of the TGFBR1 and TGFBR2 genes was therefore performed. A heterozygous mutation (c.683_685del) (p.Glu228del) in the TGFBR1 gene, which has not been reported in the literature, was identified. Genetic analysis of the parents revealed normal sequences at c.683_685 of the TGFBR1 gene (Fig. 4). Based on these results, the patient was diagnosed with LDS with a de novo mutation in the TGFBR1 gene.

At 7 years of age, valve-sparing aortic root replacement was performed because of a significantly enlarged aortic sinus. The postoperative clinical course was uneventful. After a 17 month follow-up period, a brain MRI/MRA showed no changes in the multiple 2 hyperintense lesions in both cerebral hemispheres and dilated, tortuous intracranial and vertebral arteries. In spite of his intermittent headaches, he has not developed any new neurological symptoms.
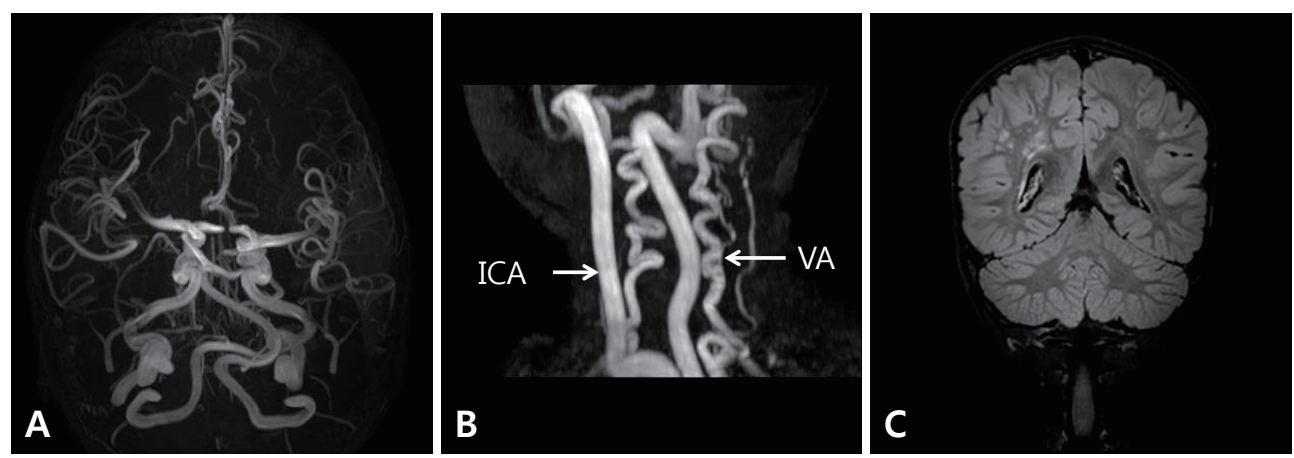

Fig. 1. Brain MRI/MRA revealed diffusely dilated, elongated, and tortuous intracranial arteries, cervical internal carotid arteries (ICA), and vertebral arteries (VA) (A, B), as well as multiple T2 hyperintense lesions in the frontal and parietal subcortical white matter $(\mathrm{C})$.
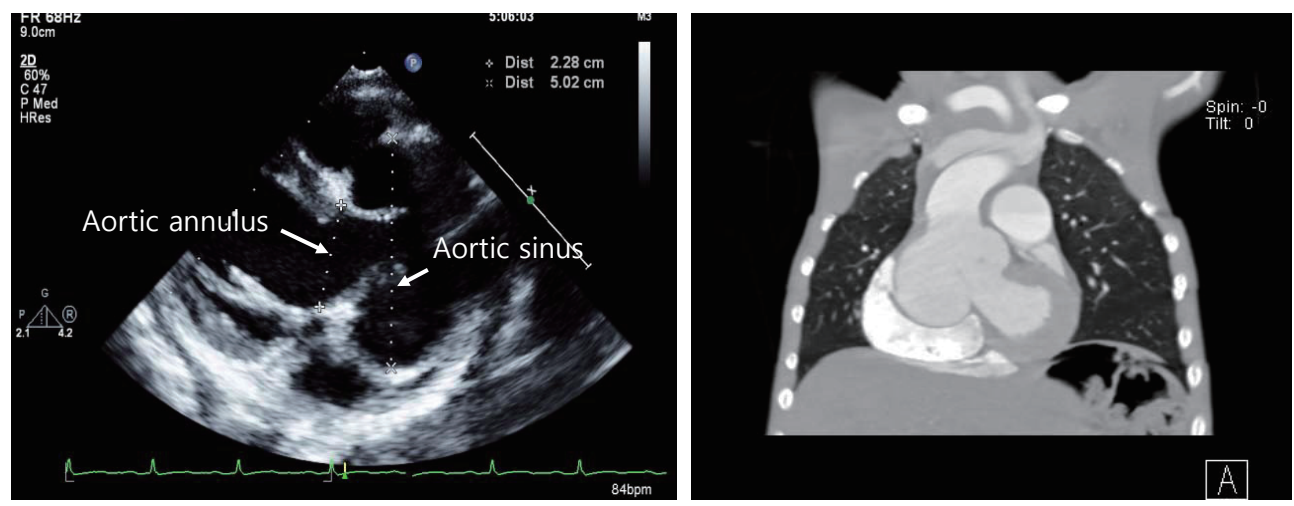

Fig. 2. Echocardiography and heart CT showed aortic root aneurysm. The aortic sinus and annulus measured $5.02 \mathrm{~cm}($ Z-value $=17.99)$ and $2.28 \mathrm{~cm}$ (Z-value=8.1), respectively. 

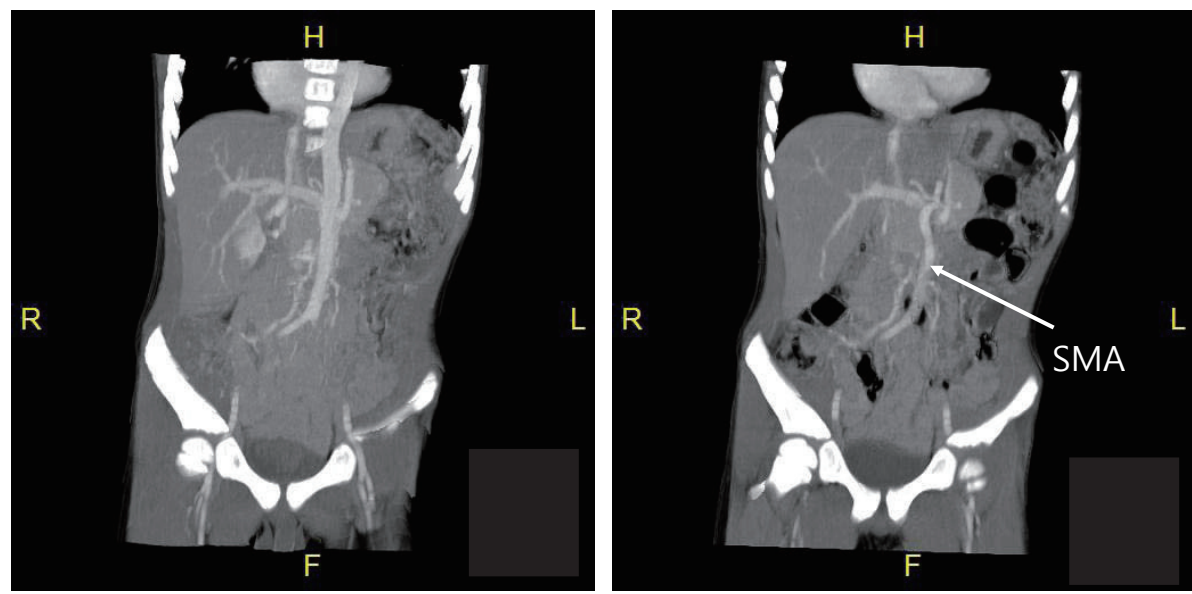

Fig. 3. Abdomen computed tomography demonstrates a prominent celiac trunk and superior mesenteric artery (SMA), and a tortuous abdominal aorta.

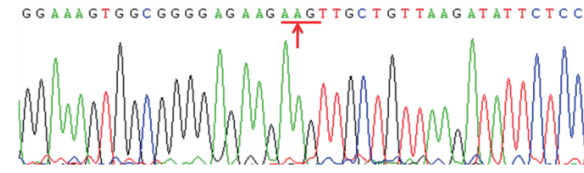

Father: Normal seq. at c.683_685(AAG)

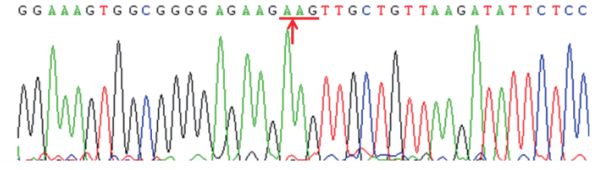

Mother: Normal seq. at c.683_685(AAG)

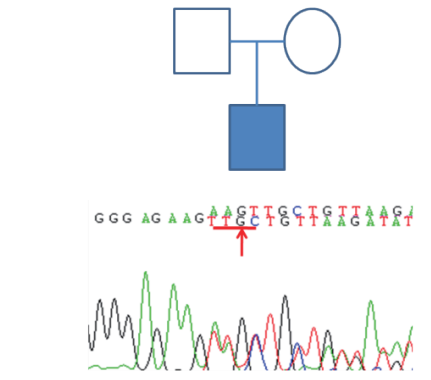

Patient: c.683 685del (p.Glu228del)

Heterozygous variation of the TGFBRI gene

Fig. 4. Pedigree of the patient's family and chromatogram of a partial DNA sequence of the TGFBR1 gene. A novel mutation (c.683_685del)(p.Glu228del) in the TGFBR1 gene was identified in the patient. Genetic analysis of his parents revealed a normal sequence at c.683_685 of the TGFBR1 gene.

\section{Discussion}

In 2005, Loeys et al., Dietz, and others described LDS as an autosomal dominant connective tissue disorder with multiple systemic involvement." LDS is characterized by the triad of arterial tortuosity and aneurysm, hypertelorism, and a bifid uvula or a cleft palate. Two subtypes of LDS have been described. LDS type 1 patients present with craniofacial manifestations, including cleft palate, craniosynostosis, or hypertelorism. Approximately threequarters of LDS patients present with typical facial dysmorphic features and are considered LDS type 1. LDS type 2 refers to patients with isolated bifid uvula and at least two of the clinical features associated with vascular Ehlers-Danlos syndrome (visceral rupture, easy bruising, wide and atrophic scars, joint laxity, and translucent and/or velvetyskin). ${ }^{2)}$

The most severe abnormality in LDS is aneurysmal dilatation of the aortic root, which is present in 98\% of LDS patients. It can result in aortic dissection and rupture, causing death at an early age. $^{2)}$ In addition to aortic root aneurysm, patients with LDS show several abnormal manifestations, including cardiac anomalies (patent ductusarteriosus, ductus aneurysm, bicuspid aortic valve, bicuspid pulmonary valve, arterial septal defects, mitral valve prolapse, and coronary artery aneurysm), craniofacial anomalies (craniosynostosis, malar hypoplasia, retrognathia and high arched palate, and dolichocephaly/scaphocephaly), and musculoskeletal anomalies (scoliosis, pectus deformity, dolichostenomelia, arachnodactyly, and camptodactyly). ${ }^{2,4,5)}$ Neuroradiologic abnormalities are also commonly seen in patients with LDS. 
Previous studies have reported neuroradiologic features such as craniosynostosis, intracranial artery tortuosity, aneurysm, dissection and stroke, Chiari 1 malformation, and hydrocephalus, accompanied by neurocognitive symptoms such as developmental delays, sensory hearing loss, and headaches. ${ }^{2,6)}$

A diagnosis of LDS is based on characteristic clinical findings and can be confirmed by molecular genetic testing of TGFBR1 and TGFBR2. LDS is caused by an autosomal dominant, heterozygous mutation of TGFBR1 or TGFBR2. Of confirmed LDS patients, 25\% have an affected parent. The remaining $75 \%$ of newly diagnosed patients present with de novo mutations. ${ }^{7)}$ Mutations of TGFBR genes are associated with increased TGF- $\beta$ signaling by nuclear accumulation of phosphorylated Smad2 and expression of TGF$\beta$ responsive gene products. As a result, increased collagen deposition and elastic disarrangements in the aortic media cause dilatation and dissection of the aortic wall in LDS. ${ }^{2,8}$

The LDS phenotype may resemble other connective tissue disorders such as Marfan syndrome (MFS), Shprintzen-Goldberg syndrome (SGS), and vascular Enlers-Danlos syndrome (EDS). MFS is caused by mutations in the FBN1 gene, which encodes fibrillin-1, and patients also present with arterial aneurysm and dissection. Whereas aneurysm and dissection in MFS are confined to the aortic root, aneurysms in LDS may involve other vessels. In LDS patients, widespread arterial tortuosity throughout the arterial tree as well as the aortic root cause earlier rupture than in MFS. Also, approximately $60 \%$ of patients with MFS develop lens dislocation, which is rarely seen in LDS. ${ }^{1,2)}$ The craniofacial abnormalities of LDS may resemble those of SGS, but cleft palate/bifid uvula and aortic/ arterial aneurysms are rare in SGS. In LDS type 2, cutaneous findings are similar to those of EDS, which is caused by mutations in COL3A1. Because neurovascular manifestations including intracranial aneurysms, carotid-cavernous fistulas, and cervical artery dissections can be seen in vascular EDS, it may be difficult to discriminate between LDS and EDS. Therefore, in individuals in which vascular EDS is suspected but COL3A1 mutations are absent, a diagnosis of LDS should be considered. ${ }^{2,9}$

The patient whom we described in this report was diagnosed with LDS because of clinical findings such as a bifid uvula, aortic aneurysm, and tortuosities of multiple arteries, and this diagnosis was confirmed by the detection of a novel mutation of TGFBR1 (c.683_685del)(p.Glu228del). This case also showed neurovascular manifestations, including dilatation and tortuosities of intracranial arteries and persistent headaches. It is the first report documenting the neurovascular manifestation of LDS in a child in Korea. The exact incidence of neurovascular manifestations in LDS patients is not clear. In a previous report, Loeys et al. noted that approximately 10\% of LDS patients had aneurysms in the arteries of the head and neck, and cerebral hemorrhage accounted for $7 \%$ of LDS deaths. ${ }^{2}$ Rodrigues et al. reported that a third of 25 LDS patients had intracranial or extracranial aneurysms, and also observed a high rate of dissection (12\%). ${ }^{6}$ Intracranial aneurysms, which carry a high risk of rupture ${ }_{1}^{5,10)}$ may cause headaches and focal neurologic symptoms. Although the mechanisms for the development of neurologic symptoms are not clear, some studies suggest a combination of the mass effect of aneurysm and the pulsatile effect of the aneurysmal sac. ${ }^{11,12)}$

LDS is an aggressive aortic and distal arterial aneurysm syndrome predisposed to dissection and rupture earlier than other connective tissue diseases; therefore, early diagnosis and appropriate intervention are essential to prevent life-threatening complications. Since neurovascular abnormalities also can be accompanied by LDS, patients with any suspicion of LDS should be evaluated by imaging modalities such as brain MRA, and appropriate interventions should be taken to prevent neurologic complications.

\section{References}

1. Loeys BL, Chen J, Neptune ER, Judge DP, Podowski M, Holm T, et al. A syndrome of altered cardiovascular, craniofacial, neurocognitive and skeletal development caused by mutations in TGFBR1 or TGFBR2. Nat Genet 2005;37:275-81.

2. Loeys BL, Schwarze U, Holm T, Callewaert BL, Thomas GH, Pannu H, et al. Aneurysm syndromes caused by mutations in the TGF-beta receptor. N Engl J Med 2006;355:788-98.

3. Williams JA, Loeys BL, Nwakanma LU, Dietz HC, Spevak PJ, Patel ND, et al. Early surgical experience with Loeys-Dietz: a new syndrome of aggressive thoracic aortic aneurysm disease. Ann Thorac Surg 2007;83: S757-63; discussion S785-90.

4. Kalra VB, Gilbert JW, Malhotra A. Loeys-Dietz syndrome: cardiovascular, neuroradiological and musculoskeletal imaging findings. Pediatr Radiol 2011;41:1495-504; quiz 1616.

5. Kilic $E_{1}$ Alanay $Y$, Utine $E$, Ozgen-Mocan B, Robinson PN, Boduroğlu K. Arterial tortuosity and aneurysm in a case of Loeys-Dietz syndrome type IB with a mutation p.R537P in the TGFBR2 gene. Turk J Pediatr 2012;54:198-202.

6. Rodrigues VJ, Elsayed S, Loeys BL, Dietz HC, Yousem DM. Neuroradiologic manifestations of Loeys-Dietz syndrome type 1. AJNR Am J Neuroradiol 2009;30:1614-9.

7. Van Hemelrijk C, Renard M, Loeys B. The Loeys-Dietz syndrome: an update for the clinician. Curr Opin Cardiol 2010;25:546-51.

8. Lindsay ME, Schepers D, Bolar NA, Doyle JJ, Gallo E, Fert-Bober J, et al. Loss-of-function mutations in TGFB2 cause a syndromic presentation 
of thoracic aortic aneurysm. Nat Genet 2012:44:922-7.

9. Zanotti G, Vricella L, Cameron D. Thoracic aortic aneurysm syndrome in children. Semin Thorac Cardiovasc Surg Pediatr Card Surg Annu 2008:11-21.

10. Brown RD. Unruptured intracranial aneurysms. Semin Neurol 2010;30: 537-44.
11. Rodriguez-Catarino $M$, Frisén L, Wikholm G, Elfverson J, Quiding $L$, Svendsen P. Internal carotid artery aneurysms, cranial nerve dysfunction and headache: the role of deformation and pulsation. Neuroradiology 2003;45:236-40.

12. Valença $M M$, Andrade-Valença LP, Martins $C$, de Fátima Vasco Aragão $\mathrm{M}$, Batista LL, Peres MF, et al. Cluster headache and intracranial aneurysm. J Headache Pain 2007:8:277-82. 